\title{
Outage and Capacity Performance Evaluation of Distributed MIMO Systems over a Composite Fading Channel
}

\author{
Wenjie Peng, Yueheng Li, Meiyan Ju, Ping Huang, Guoping Tan, and Defu Jiang \\ College of Computer and Information, Hohai University, Nanjing, Jiangsu 211100, China \\ Correspondence should be addressed to Yueheng Li; yueheng_li@hhu.edu.cn
}

Received 6 June 2014; Revised 6 September 2014; Accepted 10 September 2014; Published 29 September 2014

Academic Editor: Gerhard-Wilhelm Weber

Copyright ( 2014 Wenjie Peng et al. This is an open access article distributed under the Creative Commons Attribution License, which permits unrestricted use, distribution, and reproduction in any medium, provided the original work is properly cited.

\begin{abstract}
The exact closed-form expressions regarding the outage probability and capacity of distributed MIMO (DMIMO) systems over a composite fading channel are derived. This is achieved firstly by using a lognormal approximation to a gamma-lognormal distribution when a mobile station (MS) in the cell is in a fixed position, and the so-called maximum ratio transmission/selected combining (MRT-SC) and selected transmission/maximum ratio combining (ST-MRC) schemes are adopted in uplink and downlink, respectively. Then, based on a newly proposed nonuniform MS cell distribution model, which is more consistent with the MS cell hotspot distribution in an actual communication environment, the average outage probability and capacity formulas are further derived. Finally, the accuracy of the approximation method and the rationality of the corresponding theoretical analysis regarding the system performance are proven and illustrated by computer simulations.
\end{abstract}

\section{Introduction}

Because of the contradiction between limited spectrum resources and expanding wireless communication business needs, an increasing number of researchers are focusing on the DMIMO system, whose structures differ from ordinary systems. As it makes full use of the space microdiversity and macrodiversity [1], the DMIMO system is able to double the capacity without increasing bandwidth and it has huge advantages with regard to improving the reliability of each communication link, reducing power consumption, and expanding the signal coverage when compared with the traditional collocated MIMO (CMIMO) systems [2]. Therefore, the DMIMO system is regarded as one of the alternatives for future B4G/5G wireless communication systems [3]. In order to put it into application, the designers need to settle the challenges it poses, which include the antenna ports' location and wireless resource allocation. Moreover, as two general performance indexes, the outage probability and capacity have a very important guiding value on the optimization design of the communication system; therefore it is necessary to analyze the outage probability and capacity of DMIMO systems.

The authors [1] were the first to discuss the DMIMO's considerable advantage with regard to capacity; this was demonstrated by using the probability density function (PDF) of received signal's signal to noise ratio (SNR), which has only simulation data and not the closed-form expression. Moreover, [4] derived the expression of outage probability by considering the Nakagami- $m$ fading, path loss, and shadowing in the uplink with an MS equipped with one antenna, which differed from other multiantennae study cases. Authors in [5] studied the outage probability in a downlink with multiantennae in an MS over the Rayleigh channel. A closed-form expression of outage probability in a DMIMO over the Rayleigh channel is derived by using a Gauss random variable to approximate mutual information in [6], but the expression differs depending on the number of base stations. Instead of the Rayleigh channel in [5], authors in [7] established a composite fading channel and derived an approximate closed-form capacity expression with only one antenna at an MS.

However, all the researchers mentioned above did not take the distribution of an MS into account. This is an important issue and will be discussed in this paper as it affects the outage probability and capacity of DMIMO systems. Having studied the extant research, this paper will highlight how the composite fading channel and MS distribution 
influence the performance of DMIMO systems; furthermore, the exact closed-form expressions of outage probability and capacity will be derived by using approximate processing with multiantennae at an MS.

The remainder of this paper is organized as follows. In the next section the system model will be formulated and the approximate distribution of output SNR by replacing the gamma-lognormal distribution with a lognormal distribution will be provided. In Section 3, the outage probability and capacity of a DMIMO system are proved to be relative to the distribution of an MS, and then the average outage probability and average capacity are proposed and calculated, with the exact closed-form expressions derived. The simulation results are presented in Section 4 and conclusions are drawn in Section 5.

\section{System Model}

Consider a DMIMO system with $K$ antenna ports (AP), instead of a traditional base station, set separately in the cell, as shown in Figure 1.

All the APs equipped with one antenna are linked to the main processing unit (MPU) by an optical fiber. The MS in the cell is equipped with $L$ antennae, and the positions of the MS and the $i$ th $\mathrm{AP}$ are denoted by the rectangular coordinates $(x, y)$ and $\left(x_{i}, y_{i}\right)$, where $i=1,2, \ldots, K$.

The reason why only one MS is analyzed in the DMIMO system needs to be explained before the transmitting and receiving schemes in the uplink and downlink are introduced in the following sections. In an actual communication environment, there is always more than one user in a cell and all the users at work will transmit signals to and receive signals from the APs. Inevitably, the uplink signals will influence some APs and the downlink signals will influence other users; thus it is referred to as multiuser interference (MUI). In modem wireless communication systems such as $3 \mathrm{G}$ and $4 \mathrm{G}$, frequency division multiple access (FDMA), time division multiple access (TDMA), and code division multiple access (CDMA) techniques are often used to overcome the MUI. In FDMA and TDMA, frequency nonoverlapping subchannels and nonoverlapping time slots are assigned to each user, respectively, in order to protect them from MUI. In CDMA, the separation of each user is facilitated by the fact that each signal is spread throughout a frequency by a pseudorandom code sequence. However, the multiaccess interference (MAI) and intersymbol interference (ISI) are unavoidable when dispreading for the existence of the multipath effect. However, former researchers $[8,9]$ have proved that whether or not the interference cancellation technology is applied in CDMA, not only are the original MAI and ISI produced directly by the multipath effect, but also the residual interference that remains after applying some necessary interference suppression technologies to MAI and ISI in the receiver can be approximated to an additive Gaussian noise, which means that the MUI in CDMA can be processed as an additional background Gaussian noise imposed on the thermal noise. Indeed, the high spreading gain and interference suppression technology will overcome or ignore

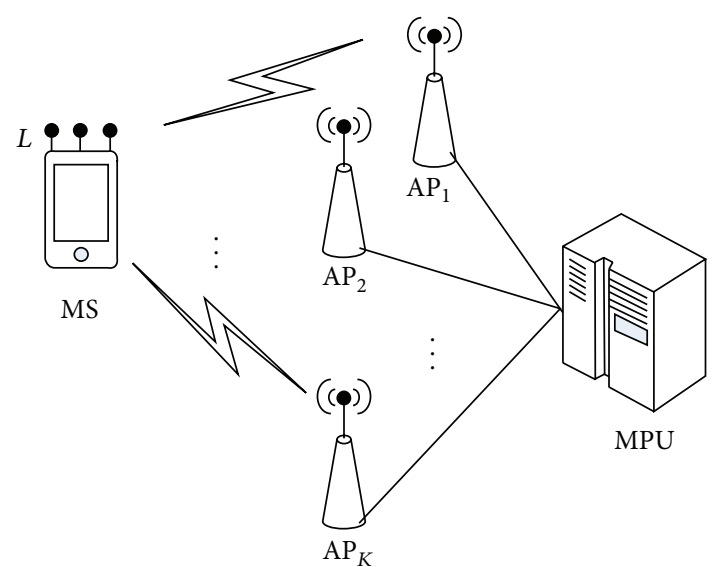

FIGURE 1: Diagram of a distributed MIMO system structure.

this additionally increased background noise, but this issue will not be discussed more since it has overstepped the main research contents of this paper. Moreover, in this way, the total MAI plus ISI or the residual interference in CDMA can be regarded as an additional background noise, which will greatly simplify or facilitate the performance analysis of this paper because the main indexing parameters, that is, the outage probability and channel capacity, are based on the output SNR of the DMIMO system.

Under these conditions, according to the classical DMIMO system, this paper takes one MS as an example to carry out an analysis. As for the uplink transmitting and receiving schemes, MRT [10] is used at the MS to maximize the output SNR, and SC is adopted to select the optimal AP. In the downlink, APs and the MS apply ST and MRC schemes, respectively.

Indeed, both the proposed MRT-SC in the uplink and the ST-MRC in the downlink apply selection diversity, which selects the AP in order to establish the communication link with the MS according to the condition of the channel between them, while, in some papers, all the APs are used to transmit and receive signals in the system [11]; in comparison, the MRT-SC and ST-MRC are simpler and can take advantage of the separate locations of the APs in the cell to reduce the average MS access distance, which lowers the transmitting and receiving power consumption [1]. Moreover, the selective schemes are helpful in minimizing the influences on the other users and intercells. At the MS, MRT and MRC are used instead of the space time block coding (STBC), which is believed to be able to improve the performance of the MIMO system and is attracting increasing attention. This is due to the fact that in a future large-scale MIMO, the application of STBC will greatly increase the decoding complexity of the system. Most importantly, [12] has proved that if, and only if, there are two transmit antennae, the system using STBC can obtain the most high output SNR, which is still less than that of the system using MRC.

Based on the above analysis, it can be concluded that the proposed MRT-SC in the uplink and ST-MRC in the downlink are capable of exploiting the potential of MIMO 
technology in outage probability and capacity and highlight the advantages of the DMIMO system.

2.1. MRT-SC in the Uplink. In the uplink, the channel is assumed to be quasistatic, and the channel coefficients are perfectly estimated at the MS. $h_{i}^{j}(j=1,2, \ldots, L)$ represents the composite channel fading between the $j$ th antenna of the MS and the $i$ th AP named as $\mathrm{AP}_{i} . E$ is the transmitting power and $x$ is the transmitted symbol with unitary power. Multiplied by the phase rotation factor $b_{i}^{j}=\left(h_{i}^{j}\right)^{*} /\left|h_{i}^{j}\right|$ in an MRT scheme, $x$ is transmitted with power $G_{i}^{j}$ :

$$
G_{i}^{j}=\frac{E \cdot\left|h_{i}^{j}\right|^{2}}{\sum_{j=1}^{L}\left|h_{i}^{j}\right|^{2}} .
$$

The signal received by $\mathrm{AP}_{i}$ can be written as

$$
\begin{aligned}
y_{i} & =\sum_{j=1}^{L} h_{i}^{j} \sqrt{G_{i}^{j}} b_{i}^{j} x+z \\
& =\sqrt{E \sum_{j=1}^{L}\left|h_{i}^{j}\right|^{2}} x+z,
\end{aligned}
$$

where $z$ is the complex white Gaussian noise with zero mean and a variance of $N_{0}$.

With the integration of Nakagami- $m$ fading, path loss, and lognormal shadowing, $h_{i}^{j}$ is modeled as

$$
h_{i}^{j}=g_{i}^{j} \sqrt{S_{i}^{j}},
$$

where $g_{i}^{j}$ represents the Nakagami- $m$ fading between the $j$ th antenna of the MS and $\mathrm{AP}_{i}$. Due to the APs being widely separated, $g_{i}^{j}(i=1,2, \ldots, K)$ is independent but not identically distributed. The MS is size limited, which means that the signals from different MS antennae suffer the same fading when transmitted to $\mathrm{AP}_{i}$. In short, $g_{i}^{j}$ follows a fast small-scale fading, that is, Nakagami- $m$ distribution [13], and the shape parameter $m_{i}$ of this distribution is influenced by the choice of AP and not the antenna of the MS.

$S_{i}^{j}$ stands for the shadowing, and the signals from the MS to $\mathrm{AP}_{i}$ undergo the same shadow fading; therefore

$$
S_{i}^{1}=S_{i}^{2}=\cdots=S_{i}^{L}=S_{i} .
$$

As it is modeled to a lognormal distribution, the PDF of $S_{i}$ can be expressed as [13]

$$
f_{S_{i}}(s)=\frac{\xi}{\sqrt{2 \pi} \sigma_{i} s} \exp \left[-\frac{\left(10 \lg s-\mu_{i}\right)^{2}}{2 \sigma_{i}^{2}}\right],
$$

where $\xi=10 / \ln 10, \sigma_{i}$ (in $\mathrm{dB}$ ) is the standard deviation of $10 \lg S_{i}$, and $\mu_{i}$ (in $\mathrm{dB}$ ) is the mean, which is determined by the path loss between the MS and $\mathrm{AP}_{i}[14] . \mu_{i}$ is modeled as

$$
\mu_{i}=10 \lg \left(\frac{d_{0}}{d_{i}}\right)^{\beta}
$$

where $\beta$ is the path loss exponent, $d_{0}$ is the reference distance, and $d_{i}$, representing the distance from $\mathrm{AP}_{i}$ to the $\mathrm{MS}$, is a function of the position of the MS:

$$
d_{i}=\sqrt{\left(x_{i}-x\right)^{2}+\left(y_{i}-y\right)^{2}} .
$$

From (1), the received $\mathrm{SNR}$ at $\mathrm{AP}_{i}$ can be expressed as

$$
\gamma_{i}=\frac{E}{N_{0}} \sum_{j=1}^{L}\left|h_{i}^{j}\right|^{2}=\sum_{j=1}^{L} \Omega_{i}\left|g_{i}^{j}\right|^{2},
$$

where $\Omega_{i}=E S_{i} / N_{0}$.

Because of $S_{i}, \Omega_{i}$ is also lognormal distributed, and the $\mathrm{PDF}$ is derived as

$$
f_{\Omega_{i}}(\omega)=\frac{\xi}{\sqrt{2 \pi} \sigma_{i} \omega} \exp \left[-\frac{\left(10 \lg \omega-\tilde{\mu}_{i}\right)^{2}}{2 \sigma_{i}^{2}}\right],
$$

where $\widetilde{\mu}_{i}=\mu_{i}+10 \lg \left(E / N_{0}\right)$ is the mean of $10 \lg \Omega_{i}$, and $\sigma_{i}^{2}$ is the variance.

In (8), $\gamma_{i}^{j}=\Omega_{i}\left|g_{i}^{j}\right|^{2}$; the conditional PDF of $\gamma_{i}^{j}$ can be expressed as

$$
f_{\gamma_{i}^{j} \mid \Omega_{i}}(r \mid w)=\frac{m_{i}^{m_{i}} r^{m_{i}-1}}{w^{m_{i}} \Gamma\left(m_{i}\right)} \exp \left(-\frac{m_{i} r}{w}\right) .
$$

According to the definition of a moment generating function (MGF), the conditional MGF of $\gamma_{i}^{j}$ is derived as [13]

$$
\begin{aligned}
M_{\gamma_{i}^{j} \mid \Omega_{i}}(p \mid \omega) & =\int_{0}^{\infty} f_{\gamma_{i}^{j} \mid \Omega_{i}}(r \mid w) e^{p r} \mathrm{~d} r \\
& =\left(1-\frac{\omega p}{m_{i}}\right)^{-m_{i}} .
\end{aligned}
$$

Due to the independence of $g_{i}^{j}(i=1,2, \ldots, K), \gamma_{i}^{j}$ is independent when $\Omega_{i}$ is known, and the conditional MGF of $\gamma_{i}^{j}$ is given by

$$
\begin{aligned}
M_{\gamma_{i} \mid \Omega_{i}}(p \mid \omega) & =\prod_{j=1}^{L} M_{\gamma_{i}^{j} \mid \Omega_{i}}(p \mid \omega) \\
& =\left(1-\frac{\omega p}{m_{i}}\right)^{-L m_{i}} .
\end{aligned}
$$

MGF is just the Laplace transform of the PDF with argument $p$ reversed in sign [13]; that is,

$$
\mathscr{L}\left[f_{\gamma_{i} \mid \Omega_{i}}(r \mid w)\right]=M_{\gamma_{i} \mid \Omega_{i}}(-p \mid \omega),
$$

where $\mathscr{L}(\cdot)$ denotes the Laplace transform. Substitute (12) into (13); by using Formula 26 in [15], Table 17.13, which is expressed as

$$
\mathscr{L}\left(x^{v-1} e^{-a x}\right)=\Gamma(v)(s+a)^{-v},
$$

the conditional PDF of $\gamma_{i}$ can be derived as

$$
f_{\gamma_{i} \mid \Omega_{i}}(r \mid w)=\frac{m_{i}^{L m_{i}} r^{L m_{i}-1}}{\omega^{L m_{i}} \Gamma\left(L m_{i}\right)} \exp \left(-\frac{m_{i} r}{\omega}\right),
$$

where $\Gamma(\cdot)$ is the gamma function. 
From (9) and (15), the PDF of $\gamma_{i}$ can be obtained as

$$
\begin{aligned}
f_{\gamma_{i}}(r)= & \int_{0}^{\infty} f_{\gamma_{i} \mid \Omega_{i}}(r \mid \omega) f_{\Omega_{i}}(\omega) \mathrm{d} \omega \\
= & \int_{0}^{\infty} \frac{m_{i}^{L m_{i}} r^{L m_{i}-1}}{\omega^{L m_{i}} \Gamma\left(L m_{i}\right)} \exp \left(-\frac{m_{i} r}{\omega}\right) \\
& \times \frac{\xi}{\sqrt{2 \pi} \sigma_{i} \omega} \exp \left[-\frac{\left(10 \lg \omega-\tilde{\mu}_{i}\right)^{2}}{2 \sigma_{i}^{2}}\right] \mathrm{d} \omega .
\end{aligned}
$$

It is known from (16) that the received $\mathrm{SNR}$ in $\mathrm{AP}_{i}$ follows a gamma-lognormal distribution [13].

The SC scheme is applied in APs by the criterion of maximizing the output SNR, which can be expressed as

$$
\gamma=\max \left\{\gamma_{1}, \gamma_{2}, \ldots, \gamma_{K}\right\}
$$

2.2. ST-MRC in the Downlink. In the downlink, all APs transmit symbol $x$ in power $E$, and the MS receives signal $\mathbf{y}_{i}$ from $\mathrm{AP}_{i}$ :

$$
\mathbf{y}_{i}=\sqrt{E}\left[h_{i}^{1}, h_{i}^{2}, \ldots, h_{i}^{L}\right]^{T} x+\mathbf{z},
$$

where $\mathbf{z}$ is a noise vector, whose entries are independent complex Gaussian random variables with zero mean and a variance of $N_{0}$.

Under the same communication environment as the uplink, by using MRC in the MS, the received SNR from $\mathrm{AP}_{i}$ is obtained as

$$
\gamma_{i}=\sum_{j=1}^{L} \frac{E\left|h_{i}^{j}\right|^{2}}{N_{0}}=\Omega_{i} \sum_{j=1}^{L}\left|g_{i}^{j}\right|^{2} .
$$

ST is adopted in APs in order to maximize the output SNR, which is expressed as

$$
\gamma=\max \left\{\gamma_{1}, \gamma_{2}, \ldots, \gamma_{K}\right\}
$$

Comparing (8) and (17) with (19) and (20), it can be concluded that when using MRT-SC and ST-MRC schemes in the uplink and downlink, respectively, the analyses on output SNR are the same.

2.3. Approximation to the PDF. In (16), the PDF of $\gamma_{i}$ is a complicated infinite integral and there is no closed-form solution, which will make it difficult to analyze the outage probability and capacity. However, Gordon proved that a gamma-lognormal distribution can be effectively approximated by a lognormal distribution [16], depending on which, the approximate PDF of $\gamma_{i}$ can be expressed as

$$
f_{\gamma_{i}}(r) \simeq \frac{\xi}{\sqrt{2 \pi} \widehat{\sigma}_{i} r} \exp \left[-\frac{\left(10 \lg r-\widehat{\mu}_{i}\right)^{2}}{2 \widehat{\sigma}_{i}^{2}}\right],
$$

where $\widehat{\mu}_{i}$ and $\widehat{\sigma}_{i}^{2}$ are the mean and variance, and they can be derived from (16) as

$$
\begin{aligned}
& \widehat{\mu}_{i}=\mu_{i}+10 \lg \left(\frac{E}{N_{0}}\right)+\xi\left[\psi\left(L m_{i}\right)-\ln \left(m_{i}\right)\right], \\
& \widehat{\sigma}_{i}=\sqrt{\sigma_{i}^{2}+\xi^{2} \zeta\left(2, L m_{i}\right)},
\end{aligned}
$$

where $\psi(\cdot)$ is the digamma function [17] and $\zeta(\cdot, \cdot)$ is the Riemann zeta function [15]. The detailed derivation can be found in Appendix A.

From (21), the cumulative distribution function (CDF) can be derived as

$$
\begin{aligned}
F_{\gamma_{i}}(X) & =\int_{0}^{X} f_{\gamma_{i}}(r) \mathrm{d} r \\
& =1-\frac{1}{2} \operatorname{erfc}\left(\frac{10 \lg X-\widehat{\mu}_{i}}{\sqrt{2} \widehat{\sigma}_{i}}\right),
\end{aligned}
$$

where $\operatorname{erfc}(\cdot)$ is the complementary error function. The detailed derivation of (23) is given in Appendix B.

According to the analyses above, the received SNR $\gamma_{i}$ is independent. From (20) and (23), the CDF of the output SNR $\gamma$ in this DMIMO system can be derived and is relative to the CDF of $\gamma_{i}$ :

$$
\begin{aligned}
F_{\gamma}(r) & =\operatorname{Pr}\{\gamma<r\} \\
& =\operatorname{Pr}\left\{\gamma_{1}<r, \gamma_{2}<r, \ldots, \gamma_{K}<r\right\} \\
& =\prod_{i=1}^{K} F_{\gamma_{i}}(r) \\
& =\prod_{i=1}^{K}\left[1-\frac{1}{2} \operatorname{erfc}\left(\frac{10 \lg r-\widehat{\mu}_{i}}{\sqrt{2} \widehat{\sigma}_{i}}\right)\right],
\end{aligned}
$$

where $\operatorname{Pr}\{\cdot\}$ denotes probability.

\section{System Performance Analyses}

3.1. Analysis at a Certain Position. It is first necessary to define the outage probability of this DMIMO system in the same way as the probability of output SNR of the system, which needs to be less than a certain value. In this paper, since the output SNR in the uplink and downlink are the same, the following equations and discussion will take the uplink as an example, without loss of generality; thus the outage probability can be expressed as

$$
P_{\text {out }}=F_{\gamma}\left(\gamma_{\text {th }}\right)=\prod_{i=1}^{K}\left[1-\frac{1}{2} \operatorname{erfc}\left(\frac{10 \lg \gamma_{\text {th }}-\widehat{\mu}_{i}}{\sqrt{2} \widehat{\sigma}_{i}}\right)\right],
$$

where $\gamma_{\text {th }}$ is the SNR threshold.

Differentiating (24) with respect to $r$ yields the PDF of $\gamma$ :

$$
\begin{aligned}
f_{\gamma}(r)=F_{\gamma}^{\prime}(r) & =\sum_{i=1}^{K}\left[f_{\gamma_{i}}(r) \prod_{j \neq i}^{K} F_{\gamma_{j}}(r)\right] \\
= & \sum_{i=1}^{K}\left\{\frac{\xi}{\sqrt{2 \pi} \widehat{\sigma}_{i} r} \exp \left[-\frac{\left(10 \lg r-\widehat{\mu}_{i}\right)^{2}}{2 \widehat{\sigma}_{i}^{2}}\right]\right. \\
& \left.\times \prod_{j \neq i}^{K}\left[1-\frac{1}{2} \operatorname{erfc}\left(\frac{10 \lg r-\widehat{\mu}_{j}}{\sqrt{2} \widehat{\sigma}_{j}}\right)\right]\right\} .
\end{aligned}
$$


With Shannon information theory, the capacity can be expressed as

$$
C=\int_{0}^{+\infty} \log _{2}(1+r) f_{\gamma}(r) \mathrm{d} r .
$$

By substituting (26) into (27), the expression of capacity in the DMIMO system can be obtained, which is complicated and has no numerical solution. Then the Gauss-Hermite integral [17] is applied to approximate it as

$$
\begin{aligned}
C \approx \frac{1}{\sqrt{\pi}} \sum_{i=1}^{K} \sum_{n=1}^{N}\{ & H_{n} \log _{2}\left(1+10^{\left(\sqrt{2} \widehat{\sigma}_{i} t_{n}+\widehat{\mu}_{i}\right) / 10}\right) \\
& \left.\times \prod_{j \neq i}^{K}\left[1-\frac{1}{2} \operatorname{erfc}\left(\frac{\sqrt{2} \widehat{\sigma}_{i} t_{n}+\widehat{\mu}_{i}-\widehat{\mu}_{j}}{\sqrt{2} \widehat{\sigma}_{j}}\right)\right]\right\},
\end{aligned}
$$

where $t_{n}$ and $H_{n}$ are, respectively, the base point and the weight point of the $N$ th order Hermite polynomial. The detailed derivation of (28) can be found in Appendix C.

So far, (25) and (28) have been derived, respectively, as closed-form expressions of the outage probability and capacity of the DMIMO system when the MS in the cell is fixed at a certain position. In this way how the communication environment affects the DMIMO system performance can be directly observed.

However, attention should be paid to the fact that the numerical values which (25) and (28) can achieve depend on the MS position when all the APs have been fixed and the networking is complete in the DMIMO system. This is because, in both of the expressions of outage probability and capacity, $\widehat{\mu}_{i}$ in fact is a function of the variable $(x, y)$, denoting the MS position. When the MS changes its coordinates, the values of outage probability and capacity will change. Therefore, (25) and (28), which represent the outage probability and capacity of the system, cannot effectively describe the physical essence of the wireless network. Even in two identical systems, the outage probability and capacity will be different with a different MS distribution if (25) and (28) are used. Therefore, in order to evaluate the DMIMO system performance, the impact of MS distribution in the cell should be considered.

\subsection{System Average Performance Analysis}

3.2.1. The MS Distribution Model. In order to make full use of the flexible networking features of the DMIMO system, this paper will apply a square, instead of the traditional circular cell planning strategy. The research results of the existing literature have shown that, with reasonable scheduling schemes, the square cell planning strategy can effectively reduce the intercell interferences caused by the MSs or base stations located in adjacent cells especially when the observed user is near the edge of a cell and thereby improve the performance of a wireless communication system [18]. In fact, there is no essential difference between a square cell and a circular cell in theoretical analysis, even though the coordinates selected are different. Compared with the polar coordinate system that is suitable for a circular cell, the Cartesian coordinate system used by the square cell in this paper is simpler and makes it more convenient to apply sector antennae (will be discussed below). This also reflects the advantage of the DMIMO in overcoming intercell interferences by using the networking flexibility from one side.

As shown in Figure 2, a new cellular cell structure based on square cell of length $D$ is proposed for following investigation. Taking the single central square cell as an example, each AP of a DMIMO system is obviously more suitable to be set at the corner of the square cell rather than to be placed at the center of the square one due to the issue of guaranteeing an effective full-cell coverage. Furthermore, in order to try to avoid the co-channel interference and improve the spectrum efficiency, four sector antennae, each of which has just 90 degrees of coverage, instead of an omnidirectional antenna should be equipped at each AP (shown as symbol $\bigcirc$ in Figure 2). This means that, from a separate square cell point of view, the square cell can be equipped by four sector antennae at its four corners following a square positioning. Similarly as in a general circle-cell DMIMO system, all the APs can be connected to one MPU by optical fiber. The MPU is responsible for commanding the APs to service MSs in the cell in an effective manner.

By using (25) and (28), the simulation results in Figure 3 are provided by Matlab in this system model, and the main simulation parameters are listed in Table 1.

Figures 3 and 4, respectively, depict how the outage probability and capacity change according to the MS position. It can be seen that, as a function of MS coordinates, the outage probability increases as the distance between the MS and the AP roughly closest increases, whereas the corresponding capacity decreases. These simulation results reveal a fact that the outage probability or capacity of DMIMO system based on square cell structure has a close relationship with the MS position or MS distribution in the cell. Therefore, it is necessary to model and consider the MS distribution in the cell in the procedure of evaluating the outage probability and capacity if the aim is to analyze the system performance comprehensively.

In previous research, the distribution of the MS is usually modeled as being uniform, which means that the probability of the MS being in any position is equal. In the square cell proposed in this paper, the PDF of uniform distribution can be expressed as

$$
f(x, y)=\frac{1}{D^{2}} .
$$

However, in an actual communication environment, the real MS distribution is far more complicated than a uniform distribution. In general, the geographic distribution of an MS is unpredictable, but it is a feature of network planning that network operators prefer setting up APs in a location where the MS distribution is denser, or, from another perspective, there are more MSs around the APs. In fact, hotspots do exist in real life, such as a supermarket where there are more people gathered than in other places. In this paper, since the location positioning of the four sector antennae have been 
TABLE 1: Simulation parameters.

\begin{tabular}{lc}
\hline Parameter & Value \\
\hline Square cell length, $D$ & $500 \mathrm{~m}$ \\
Number of MS antennae, $L$ & 2 \\
Nakagami parameter, $m_{i}$ & 1 \\
Reference distance, $d_{0}$ & $40 \mathrm{~m}$ \\
Path loss exponent, $\beta$ & 4 \\
Standard deviation of shadowing, $\sigma$ & $8 \mathrm{~dB}$ \\
SNR threshold, $\gamma_{\text {th }}$ & $0 \mathrm{~dB}$ \\
\hline
\end{tabular}

analyzed above and fixed, to simulate the scenario of the real communication environment with hotspots, it is assumed that the 4 quarter-circle regions of radius $r$ (see Figure 2), which take the corresponding APs located at the corner of the square cell as the centers, are hotspots carrying more MSs per unit area than the rest. If one considers the single square cell in the center of Figure 2, Region 1 consisting of 4 quarter circles covered by 4 sector antennae is denoted by $A_{1}$, in which the MS distribution density is higher than that in Region 2 denoted by $A_{2}$ in the square cell. Therefore the PDF of this MS distribution model in a single square cell can be expressed as

$$
f(x, y)= \begin{cases}\frac{\lambda}{\pi r^{2}}, & (x, y) \in A_{1}, \\ \frac{1-\lambda}{D^{2}-\pi r^{2}}, & (x, y) \in A_{2}\end{cases}
$$

where $\lambda \in[0,1]$ denotes the probability of the MS being in Region 1. According to the analyses above, in order for Region 1 to be considered as a hotspot, $\lambda>\pi r^{2} / D^{2}$ should be satisfied. Obviously, when $\lambda=\pi r^{2} / D^{2}$, the proposed nonuniform distribution model will reduce to a general uniform distribution. Apart from the parameter $\lambda$, the radius $r$ of the circle is also variable. By changing the values of $\lambda$ and $r$, different MS distributions can be obtained, which indicates the generality and flexibility of the proposed MS nonuniform distribution model.

3.2.2. Average Outage Probability and Capacity. It has been proved that (25) and (28), which were derived in Section 3.1, stand for the outage probability and capacity of the DMIMO system when the MS in the cell is fixed at certain position. Clearly, it is one-sided to only evaluate the system with these two expressions. Therefore, it is reasonable to take the MS distribution into account, and thus the average outage probability and average capacity of the DMIMO system are proposed. These estimate the probability-weighted outage probability and capacity of all MSs at different locations in order to describe the average performance of the whole DMIMO system.

Assume that $f(x, y)$ is the PDF used to describe the MS distribution of any kind; then the average outage probability
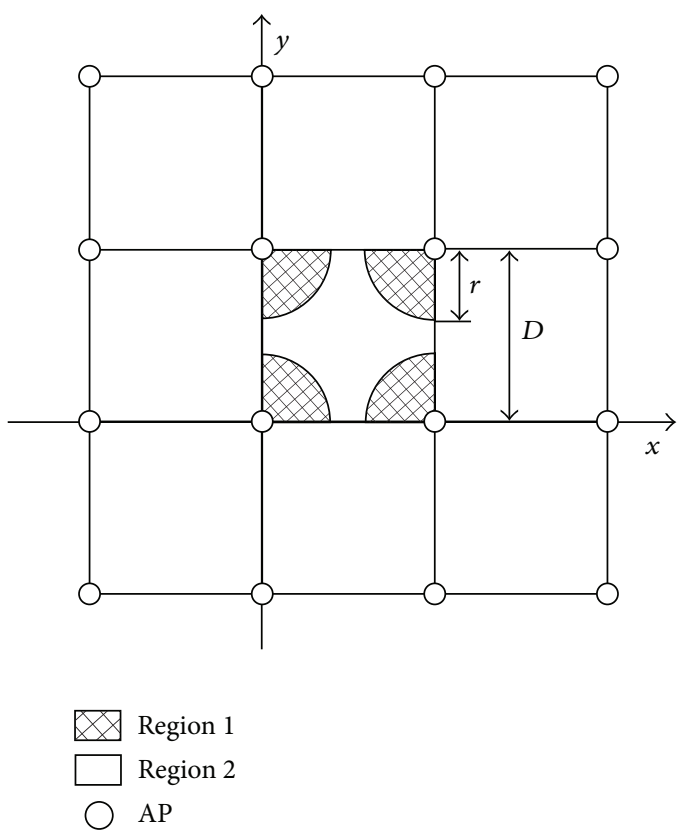

FIGURE 2: Diagram of the MS distribution.

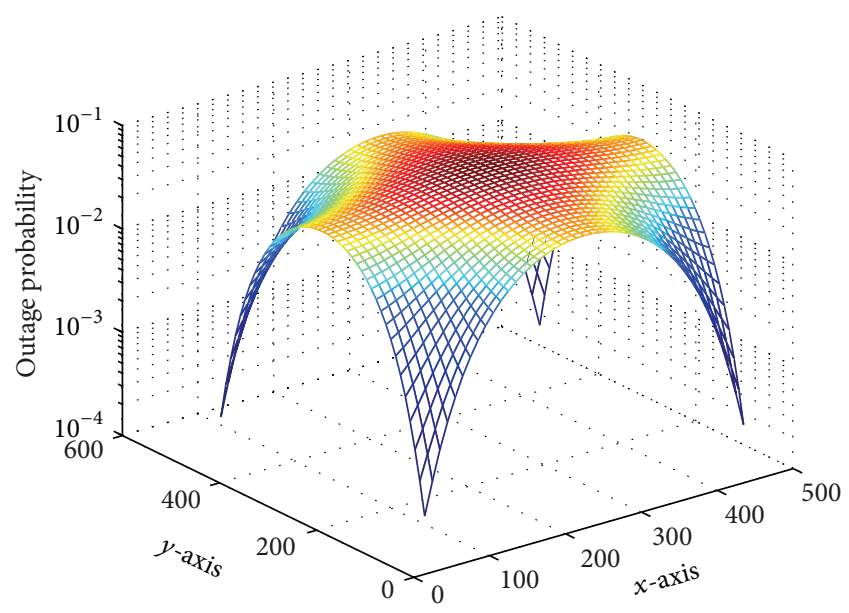

FIGURE 3: Outage probability according to the MS position.

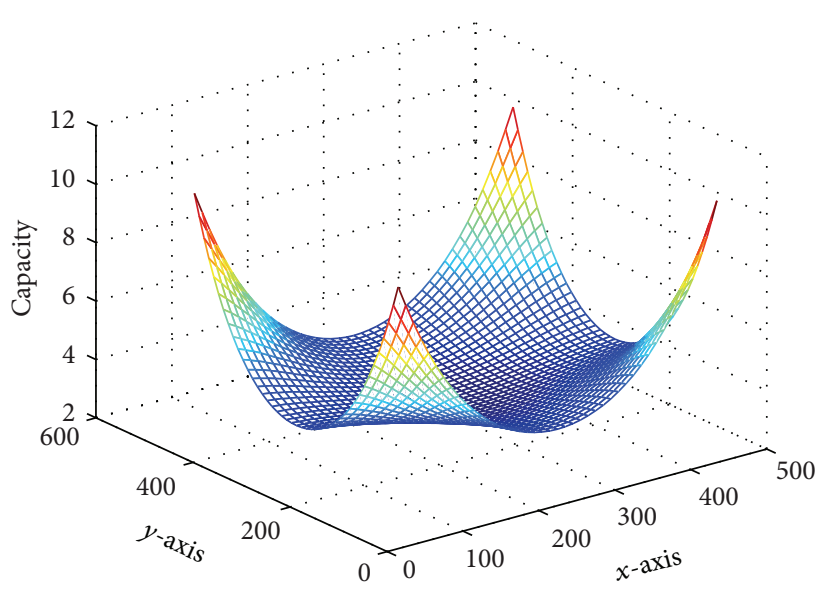

FIgURE 4: Capacity according to the MS position. 
of the DMIMO system can be ascertained by calculating the mathematical expectation of (25) as

$$
\begin{aligned}
\bar{P}_{\text {out }} & =E_{(x, y)}\left\{P_{\text {out }}\right\} \\
& =\iint_{0}^{D} P_{\text {out }}(x, y) f(x, y) \mathrm{d} x \mathrm{~d} y .
\end{aligned}
$$

Moreover the average capacity is derived by calculating the mathematical expectation of (28) as

$$
\begin{aligned}
\bar{C} & =E_{(x, y)}\{C\} \\
& =\iint_{0}^{D} C(x, y) f(x, y) \mathrm{d} x \mathrm{~d} y .
\end{aligned}
$$

Sometimes $f(x, y)$, which represents the MS distribution, will be so complicated that there may be no closed-form solution for (31) or (32). In such a situation, composite Simpson's rule [19] is introduced in order to solve the double integrations, and the final average outage probability can be expressed as

$$
\bar{P}_{\text {out }} \simeq \frac{h k}{9} \sum_{p=0}^{P} \sum_{q=0}^{Q}\left[a_{p, q} P_{\text {out }}\left(x_{p}, y_{q}\right) f\left(x_{p}, y_{q}\right)\right] .
$$

Additionally the average capacity is given as

$$
\bar{C} \simeq \frac{h k}{9} \sum_{p=0}^{P} \sum_{q=0}^{Q}\left[a_{p, q} C\left(x_{p}, y_{q}\right) f\left(x_{p}, y_{q}\right)\right]
$$

where $P$ and $Q$ are both even integers, $h=D / P, k=D / Q$, $x_{p}=p h$, and $y_{q}=q k$. The weight factor $a_{p, q}$ is the element of matrix $\mathbf{A}$, in the $(p+1)$ th row and the $(q+1)$ th column, and the weight matrix $\mathbf{A}$ is given as

$$
\begin{aligned}
\mathbf{A}= & \underbrace{\left[\begin{array}{llllllll}
1 & 4 & 2 & 4 & \cdots & 2 & 4 & 1
\end{array}\right]^{T}}_{P+1} \\
& \otimes \underbrace{\left[\begin{array}{llllllll}
1 & 4 & 2 & 4 & \cdots & 2 & 4 & 1
\end{array}\right]}_{Q+1},
\end{aligned}
$$

where $[\cdot]^{T}$ denotes the matrix transpose and $\otimes$ denotes the Kronecker product.

\section{Simulation Results}

In this section, the theoretical results analyzed in the above Section 3 are compared with those of the Monte-Carlo simulation in order to verify the accuracy of the average outage probability expression and average capacity expression. Furthermore, the impact of the Nakagami parameter, the number of MS antennae, the path loss exponent, and the MS distribution in the cell on final system performance are discussed with the aim of confirming the rationality of the analyses in this paper. The main parameters used in the simulation are listed in Tables 1 and 2.

Figures 5-7 plot the theoretical and computer simulation values of the DMIMO system average outage probability
TABLE 2: Simulation parameters.

\begin{tabular}{lc}
\hline Parameter & Value \\
\hline Distribution parameter, $\lambda$ & 0.7 \\
Radius of hotspot, $r$ & $350 / \sqrt{\pi}$ \\
Order of Hermite polynomial, $N$ & 20 \\
Composite Simpson's rule parameter, $P, Q$ & 20 \\
\hline
\end{tabular}

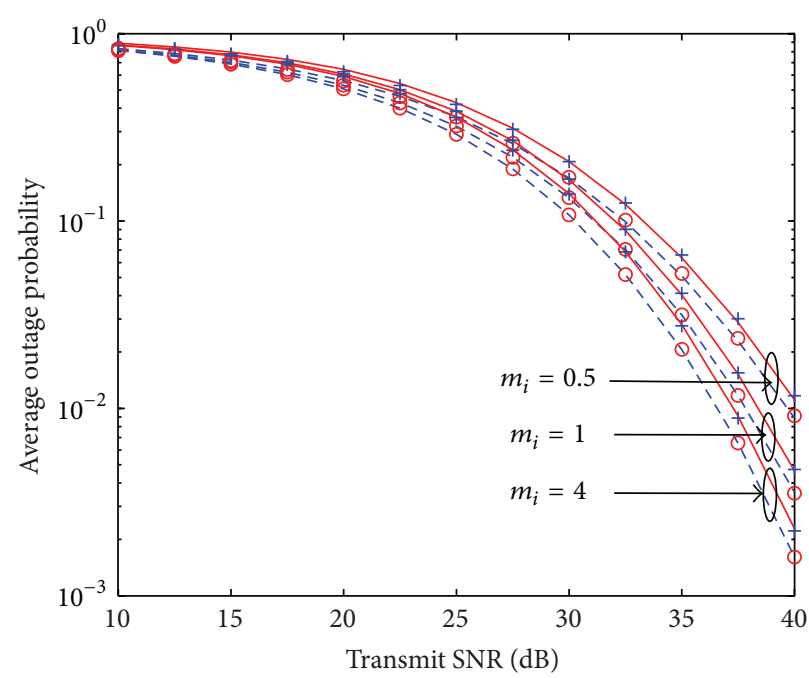

$$
\begin{array}{ccc}
\text { - Uniform distribution } & \text { (simulation) } \\
+\quad \begin{array}{c}
\text { Uniform distribution } \\
\text { (theory) }
\end{array} & \bigcirc & \begin{array}{c}
\text { Nonuniform distribution } \\
\text { (simulation) }
\end{array} \\
\text { (theory) }
\end{array}
$$

FIGURE 5: Average outage probability versus transmit SNR with different Nakagami parameters and MS distributions.

versus the transmit SNR with a different MS distribution in different scenarios. Additionally Figures 8-10 illustrate the system average capacity as a function of the transmit SNR. All the figures show that, as the transmit SNR increases, the average outage probability decreases and the average capacity increases.

Specifically, Figures 5 and 8 depict the impact of microscopic fading on DMIMO system's performances. The severity of microscopic fading decreases with the increase of $m_{i}$; thus the bigger the values of $m_{i}$ are, the better the wireless channel performs. Consequently, the output SNR of the DMIMO system improves, and then the average outage probability decreases, and average capacity increases. Figures 6 and 9 , respectively, plot the average outage probability and average capacity with different numbers of MS antennae. More antennae provide more space diversity gain, which improves the average outage and capacity performances of the system. Figures 7 and 10 show the average outage probability and average capacity with various path loss exponents. A lower path loss can be achieved by decreasing the path loss exponent; subsequently better average outage and capacity performances are achieved.

It can be clearly seen from the curves in these figures that, with the MS in the cell following the proposed nonuniform 


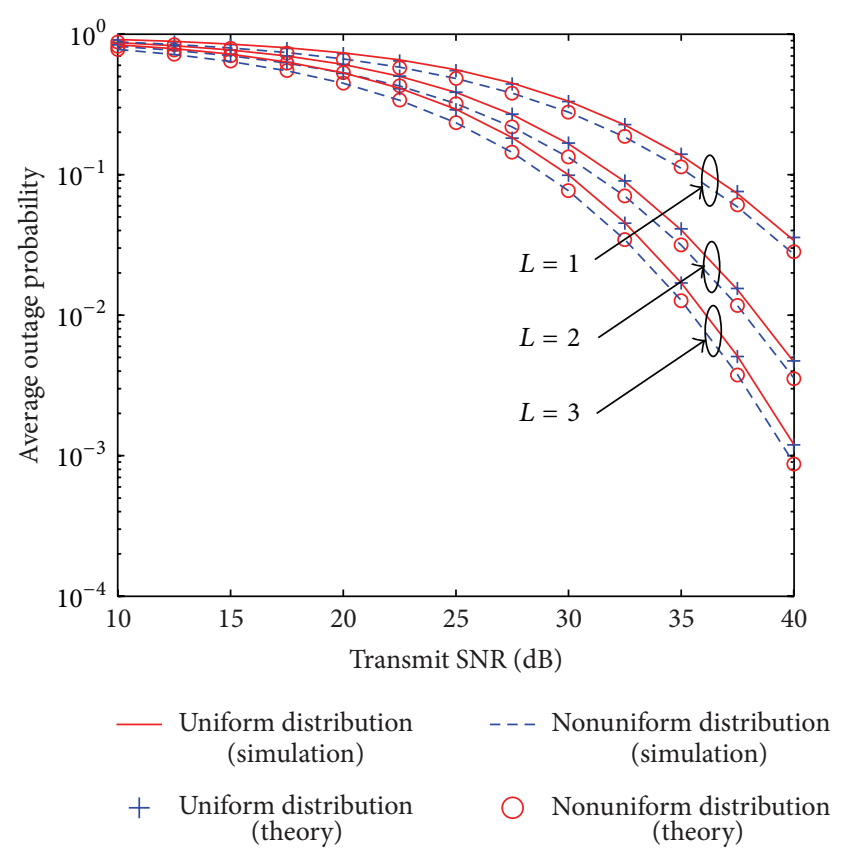

FIGURE 6: Average outage probability versus transmit SNR with different numbers of MS antennae and MS distributions.

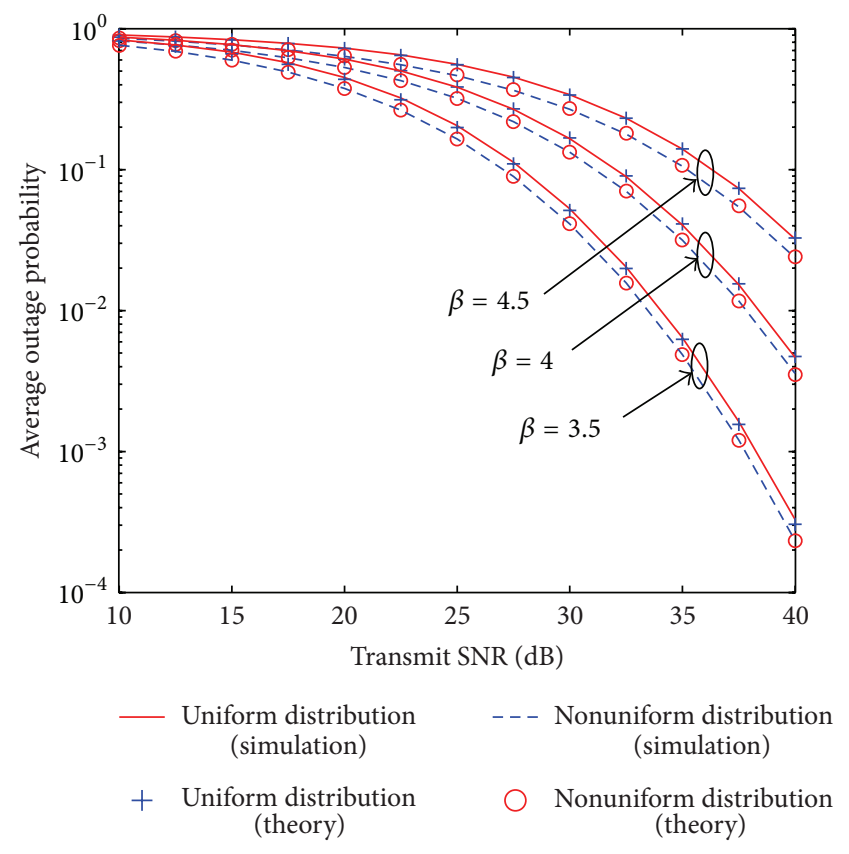

FIGURE 7: Average outage probability versus transmit SNR with different path loss exponents and MS distributions.

distribution, the DMIMO system performs better in both outage probability and capacity. This fact confirms the rationality of the outage probability and capacity analyses of the DMIMO system in this paper and indicates the importance of modeling the MS distribution correctly. At the same time, regardless of the channel fading, the MS distribution, and the number of MS antennae, the differences between

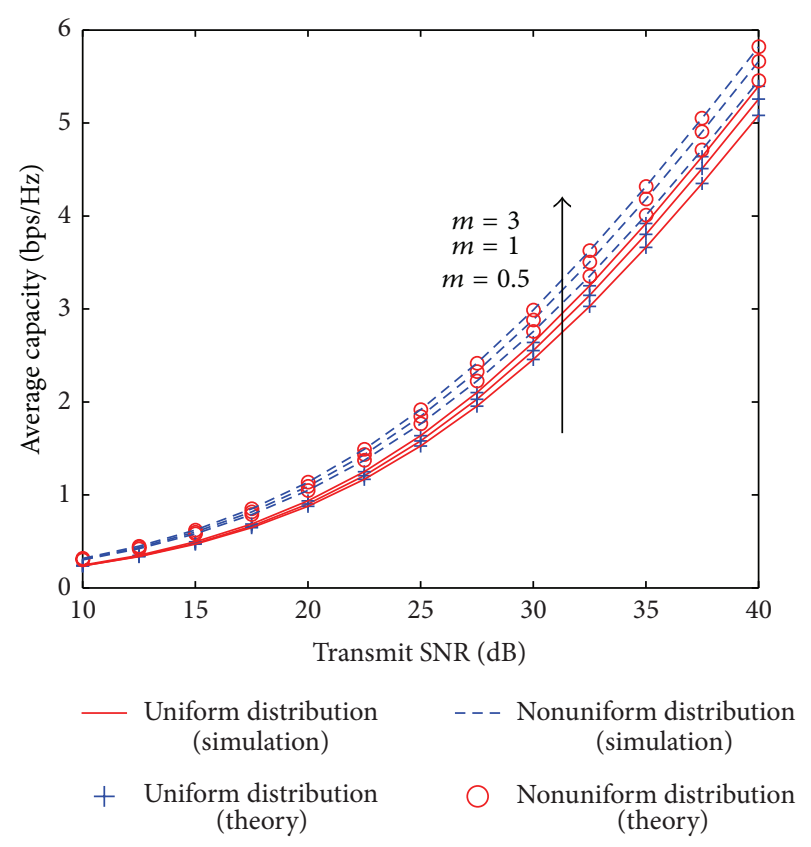

FIGURE 8: Average capacity versus transmit SNR with different Nakagami parameters and MS distributions.

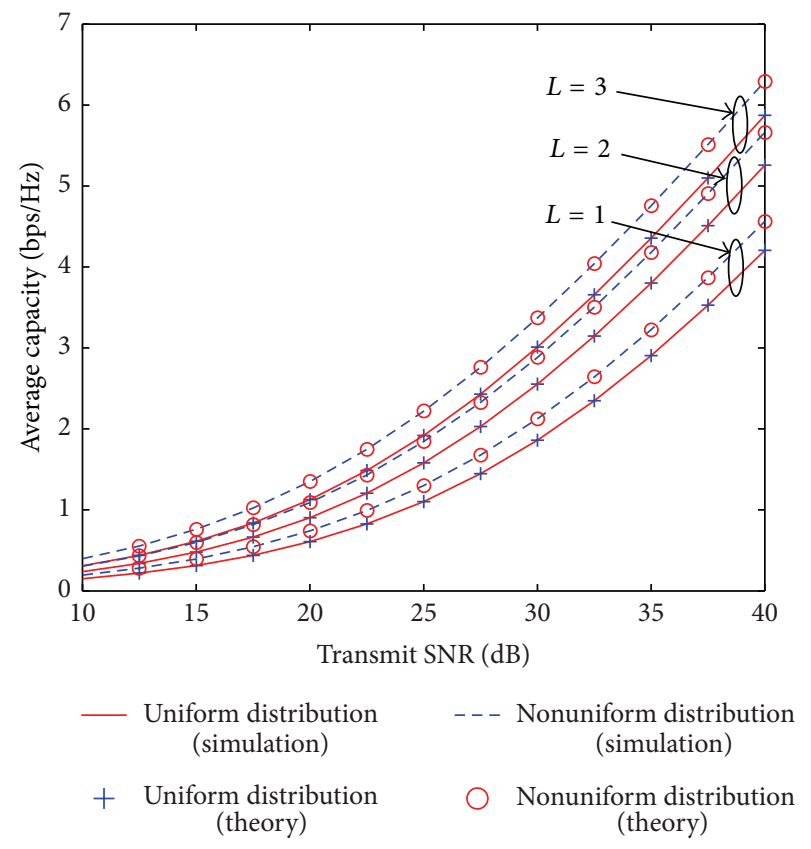

FIGURE 9: Average capacity versus transmit SNR with different numbers of MS antennae and MS distributions.

the theoretical results and simulation results are always less than $4 \%$, which is small enough to be ignored. Moreover, [20] has shown that when $m_{i}=1$ the lognormal approximation to gamma-lognormal is valid for $\sigma>6 \mathrm{~dB}$, or when $m_{i}>2$ the approximation is valid for all ranges of $\sigma$. Clearly, the value ranges fit well with the actual communication environment. In summary therefore, all the theoretical analyses and simulation results prove that the approximation used in this paper 


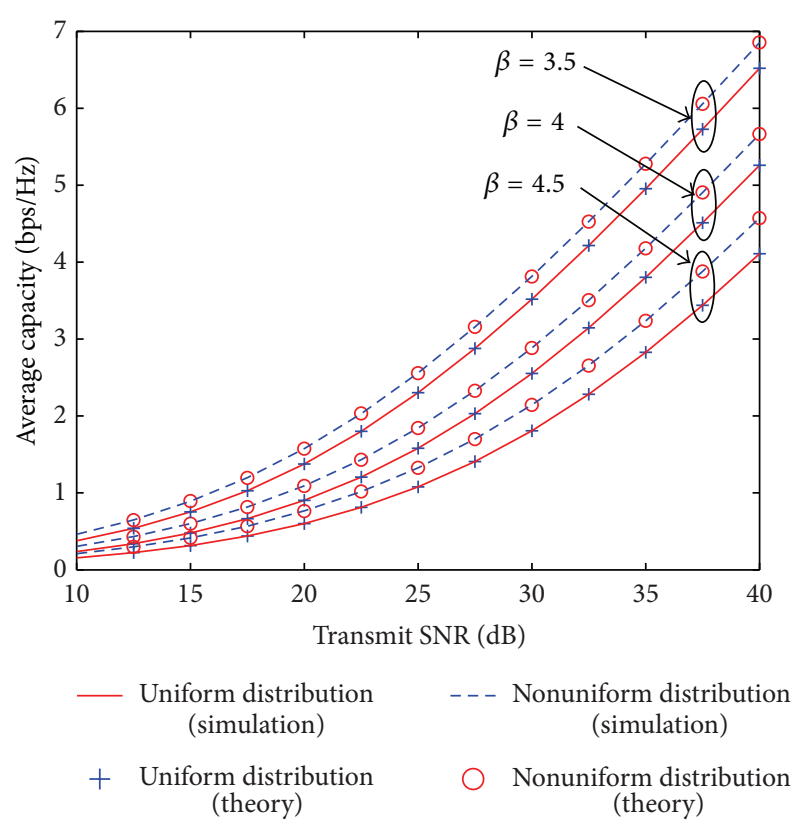

FIGURE 10: Average capacity versus transmit SNR with different path loss exponents and MS distributions.

is accurate and feasible, and the expressions of average outage probability and average capacity can accurately approximate the realistic performances of the system relatively quickly.

\section{Conclusions and Future Work}

This paper investigated the outage probability and capacity of the DMIMO system. Based on the composite fading channel integrating with Nakagami- $m$ fading, path loss, and shadow fading, the MRT-SC and ST-MRC were applied in order to maximize the output SNR in the uplink and downlink, respectively. Subsequently the exact closed-form expressions of outage probability and capacity derived when an MS is fixed at certain position were proved to be one-sided when evaluating DMIMO system performances. Therefore, taking the MS distribution into account, the average outage probability and average capacity were proposed, and the exact closed-form expressions were derived by the use of approximations. Furthermore, a newly proposed nonuniform MS cell distribution model, which is more consistent with the MS cell hotspot distribution in an actual communication environment, was applied to the expressions. Then the simulation results were obtained by using these expressions to verify the accuracy of the approximation method and the rationality of the corresponding theoretical analysis regarding the system outage probability and capacity. Furthermore, the closed-form expressions derived can precisely evaluate the effect of the channel fading, the number of antennae, and the distribution of the MS in the cell. The differences of outage probability and capacity caused by the different MS distribution models demonstrate the rationality of the analysis regarding the MS distribution and the importance of modelling the MS distribution accurately.
Besides the work presented in this paper, there are still some valuable topics which need to be further paid attention to and studied by interested researchers. First of all, since the symbol error rate (SER) is another key factor for evaluating the system performance, based on some necessary modulation and coding schemes, the SER of DMIMO system should be considered thoroughly, and similarly, its relative close-form expressions should be deduced by some possible approximations. Next, comparing with the DMIMO system built in a single cell, a multicell structure is obviously more complete and consistent with an actual working environment and is, however, more complex due to the unavoidable intercell interferences; this means that the performance analysis of a DMIMO system should be extended to a multicell situation in the future especially when the APs are equipped with directional antennae. Finally, since all the analyses on the outage, channel capacity, and SER aim to service for obtaining the corresponding optimum DMIMO system performance and the optimal values are closely related to some critical DMIMO system parameters, for example, the placement position of AP, MS distribution in the cell, transmit power, and spectrum resources allocation, and so forth, the final job should focus on modeling and achieving the desirable performance and parameter optimization strategy for the DMIMO system by using some advanced optimization algorithms.

\section{Appendices}

\section{A. Derivation of (22)}

$\widehat{\mu}_{i}$ can be derived by calculating the mathematical expectation of $10 \lg \gamma_{i}$; that is,

$$
\begin{aligned}
\widehat{\mu}_{i}= & E\left[10 \lg \gamma_{i}\right]=\int_{0}^{\infty} 10 \lg (r) f_{\gamma_{i}}(r) \mathrm{d} r \\
= & \frac{10 \xi m_{i}^{L m_{i}}}{\sqrt{2 \pi} \sigma_{i} \Gamma\left(L m_{i}\right)} \\
& \times \int_{0}^{\infty}\left\{\frac{1}{\omega^{L m_{i}+1}} \exp \left[-\frac{\left(10 \lg \omega-\widetilde{\mu}_{i}\right)^{2}}{2 \sigma_{i}^{2}}\right]\right. \\
& \left.\quad \times\left[\int_{0}^{\infty} \lg (r) r^{L m_{i}-1} \exp \left(-\frac{m_{i} r}{\omega}\right) \mathrm{d} r\right]\right\} \mathrm{d} \omega .
\end{aligned}
$$

Utilize Equation 4.352.1 in [15] given as

$$
\int_{0}^{\infty} x^{v-1} e^{-\mu x} \ln x \mathrm{~d} x=\frac{1}{\mu^{v}} \Gamma(v)[\psi(v)-\ln \mu] ;
$$

it can be ascertained from (A.1) that

$$
\begin{aligned}
\int_{0}^{\infty} \lg (r) r^{L m_{i}-1} \exp \left(-\frac{m_{i} r}{\omega}\right) \mathrm{d} r \\
\quad=\frac{w^{L m_{i}} \Gamma\left(L m_{i}\right)}{m_{i}^{L m_{i}} \ln 10}\left[\psi\left(L m_{i}\right)-\ln \left(\frac{m_{i}}{\omega}\right)\right] .
\end{aligned}
$$


Substitute (A.3) into (A.1) and denote $x=10 \lg \omega ; \widehat{\mu}_{i}$ can be expressed as

$$
\begin{aligned}
\widehat{\mu}_{i}= & \frac{\xi}{\sqrt{2 \pi} \sigma_{i}} \int_{0}^{\infty}\left[\psi\left(L m_{i}\right)-\ln \left(m_{i}\right)+\frac{x}{\xi}\right] e^{-\left(x-\widetilde{\mu}_{i}\right)^{2} / 2 \sigma_{i}^{2}} \mathrm{~d} x \\
= & \frac{\xi}{\sqrt{2 \pi} \sigma_{i}} \\
& \quad \quad\left\{\int_{0}^{\infty} \frac{x}{\xi} \exp \left[-\frac{\left(x-\tilde{\mu}_{i}\right)^{2}}{2 \sigma_{i}^{2}}\right] \mathrm{d} x\right. \\
& \left.\quad+\left[\psi\left(L m_{i}\right)-\ln \left(m_{i}\right)\right] \int_{0}^{\infty} \exp \left[-\frac{\left(x-\widetilde{\mu}_{i}\right)^{2}}{2 \sigma_{i}^{2}}\right] \mathrm{d} x\right\} \\
= & \widetilde{\mu}_{i}+\xi\left[\psi\left(L m_{i}\right)-\ln \left(m_{i}\right)\right] \\
= & \mu_{i}+10 \lg \left(\frac{E}{N_{0}}\right)+\xi\left[\psi\left(L m_{i}\right)-\ln \left(m_{i}\right)\right] .
\end{aligned}
$$

In order to obtain the variance after approximation, it is first necessary to calculate the second moment of $10 \lg \gamma_{i}$ :

$$
\begin{aligned}
E[ & \left.\left(10 \lg \gamma_{i}\right)^{2}\right] \\
= & \int_{0}^{\infty}(10 \lg r)^{2} f_{\gamma_{i}}(r) \mathrm{d} r \\
= & \frac{\xi m_{i}^{L m_{i}}}{\sqrt{2 \pi} \sigma_{i} \Gamma\left(L m_{i}\right)} \\
& \times \int_{0}^{\infty}\left\{\frac{1}{\omega^{L m_{i}+1}} \exp \left[-\frac{\left(10 \lg \omega-\tilde{\mu}_{i}\right)^{2}}{2 \sigma_{i}^{2}}\right]\right. \\
& \left.\quad \times\left[\int_{0}^{\infty}[10 \lg (r)]^{2} r^{L m_{i}-1} \exp \left(-\frac{m_{i} r}{\omega}\right) \mathrm{d} r\right]\right\} \mathrm{d} \omega .
\end{aligned}
$$

Utilize Equation 4.358.2 in [15] expressed as

$$
\int_{0}^{\infty} x^{v-1} e^{-\mu x}(\ln x)^{2} \mathrm{~d} x=\frac{\Gamma(v)}{\mu^{v}}\left\{[\psi(v)-\ln \mu]^{2}+\zeta(2, v)\right\}
$$

it can be ascertained from (A.5) that

$$
\begin{aligned}
\int_{0}^{\infty} & {[10 \lg (r)]^{2} r^{L m_{i}-1} \exp \left(-\frac{m_{i} r}{\omega}\right) \mathrm{d} r } \\
= & \xi^{2}\left(\frac{w}{m_{i}}\right)^{L m_{i}} \Gamma\left(L m_{i}\right) \\
& \times\left\{\zeta\left(2, L m_{i}\right)+\left[\psi\left(L m_{i}\right)-\ln \left(m_{i}\right)+\ln (w)\right]^{2}\right\} .
\end{aligned}
$$

Substitute (A.7) into (A.5) and denote $x=10 \lg \omega$; the second moment of $10 \lg \gamma_{i}$ is given as

$$
\begin{aligned}
E\left[\left(10 \lg \gamma_{i}\right)^{2}\right]= & \mu_{i}+\sigma_{i}^{2}+\xi^{2} \zeta\left(2, L m_{i}\right) \\
& +\xi\left[\psi\left(L m_{i}\right)-\ln \left(m_{i}\right)+\ln \left(\frac{E}{N_{0}}\right)\right] .
\end{aligned}
$$

Then, $\widehat{\sigma}_{i}$ is derived from (A.4) and (A.8) as

$$
\begin{aligned}
\widehat{\sigma}_{i} & =\sqrt{E\left[\left(10 \lg \gamma_{i}\right)^{2}\right]-\widehat{\mu}_{i}^{2}} \\
& =\sqrt{\sigma_{i}^{2}+\xi^{2} \zeta\left(2, L m_{i}\right)} .
\end{aligned}
$$

\section{B. Derivation of (23)}

The CDF of $\gamma_{i}$ can be derived from (21) as

$$
\begin{aligned}
F_{\gamma_{i}}(X) & =\int_{0}^{X} f_{\gamma_{i}}(r) \mathrm{d} r \\
& =\int_{0}^{X} \frac{\xi}{\sqrt{2 \pi} \widehat{\sigma}_{i} r} \exp \left[-\frac{\left(10 \lg r-\widehat{\mu}_{i}\right)^{2}}{2 \widehat{\sigma}_{i}^{2}}\right] \mathrm{d} r .
\end{aligned}
$$

Denote $t=\left(10 \lg r-\widehat{\mu}_{i}\right) / \sqrt{2} \widehat{\sigma}_{i}$; then the CDF can be expressed as

$$
\begin{aligned}
F_{\gamma_{i}}(X) & =\int_{-\infty}^{\left(10 \lg X-\widehat{\mu}_{i}\right) / \sqrt{2} \widehat{\sigma}_{i}} \frac{1}{\sqrt{\pi}} \exp \left(-t^{2}\right) \mathrm{d} t \\
& =1-\int_{\left(10 \lg X-\widehat{\mu}_{i}\right) / \sqrt{2} \widehat{\sigma}_{i}}^{+\infty} \frac{1}{\sqrt{\pi}} \exp \left(-t^{2}\right) \mathrm{d} t \\
& =1-\frac{1}{2} \operatorname{erfc}\left(\frac{10 \lg X-\widehat{\mu}_{i}}{\sqrt{2} \widehat{\sigma}_{i}}\right),
\end{aligned}
$$

where $\operatorname{erfc}(x)$ is the complementary error function defined as

$$
\operatorname{erfc}(x)=\frac{2}{\sqrt{\pi}} \int_{x}^{\infty} e^{-t^{2} / 2} \mathrm{~d} t
$$

\section{Derivation of (28)}

The capacity is defined as

$$
\begin{aligned}
& C=\int_{0}^{+\infty} \log _{2}(1+r) f_{\gamma}(r) \mathrm{d} r \\
&=\int_{0}^{+\infty} \log _{2}(1+r) \\
& \times \sum_{i=1}^{K}\left\{\frac{\xi}{\sqrt{2 \pi} \widehat{\sigma}_{i} r} e^{-\left(10 \lg r-\widehat{\mu}_{i}\right)^{2} / 2 \widehat{\sigma}_{i}^{2}}\right. \\
&\left.\times \prod_{j \neq i}^{K}\left[1-\frac{1}{2} \operatorname{erfc}\left(\frac{10 \lg r-\widehat{\mu}_{j}}{\sqrt{2} \widehat{\sigma}_{j}}\right)\right]\right\} \mathrm{d} r .
\end{aligned}
$$


Denoting $x=\left(10 \lg r-\widehat{\mu}_{i}\right) /\left(\sqrt{2} \widehat{\sigma}_{i}\right)$ and making use of the Gauss-Hermite integral [17] defined as

$$
\int_{-\infty}^{+\infty} e^{-x^{2}} f(x) \mathrm{d} x \approx \sum_{n=1}^{N} H_{n} f\left(t_{n}\right),
$$

the capacity can be expressed as

$$
\begin{aligned}
& C=\frac{1}{\sqrt{\pi}} \\
& \times \sum_{i=1}^{K} \int_{-\infty}^{+\infty}\left\{e^{-r^{2}} \log _{2}\left(1+10^{\left(\sqrt{2} \widehat{\sigma}_{i} r+\widehat{\mu}_{i}\right) / 10}\right)\right. \\
& \left.\times \prod_{j \neq i}^{K}\left[1-\frac{1}{2} \operatorname{erfc}\left(\frac{\sqrt{2} \widehat{\sigma}_{i} r+\widehat{\mu}_{i}-\widehat{\mu}_{j}}{\sqrt{2} \widehat{\sigma}_{j}}\right)\right]\right\} \mathrm{d} r \\
& \approx \frac{1}{\sqrt{\pi}} \sum_{i=1}^{K} \sum_{n=1}^{N}\left\{H_{n} \log _{2}\left(1+10^{\left(\sqrt{2} \widehat{\sigma}_{i} t_{n}+\widehat{\mu}_{i}\right) / 10}\right)\right. \\
& \left.\times \prod_{j \neq i}^{K}\left[1-\frac{1}{2} \operatorname{erfc}\left(\frac{\sqrt{2} \widehat{\sigma}_{i} t_{n}+\widehat{\mu}_{i}-\widehat{\mu}_{j}}{\sqrt{2} \widehat{\sigma}_{j}}\right)\right]\right\},
\end{aligned}
$$

where the base points $t_{n}$ and weight point $H_{n}$ are listed in Table 25.10 of [17].

\section{Conflict of Interests}

The authors declare that there is no conflict of interests regarding the publication of this paper.

\section{Acknowledgments}

The authors would like to express their thanks to the anonymous reviewers and the editor for their careful reviews and valuable suggestions to improve the quality of the paper. This work has been supported by the College Industrialization Project of Jiangsu Province (JHB2012-4).

\section{References}

[1] R. Wonil, High performance distributed antenna cellular networks [Ph.D. thesis], Stanford University, Palo Alto, Calif, USA, 2003.

[2] H. Hu, Y. Zhang, and J. Luo, Distributed Antenna Systems: Open Architecture for Future Wireless Communications, Auerbach Publications, CRC Press, Boca Raton, Fla, USA, 2007.

[3] S. Zhou, M. Zhao, X. Xu, J. Wang, and Y. Yao, "Distributed wireless communication system: a new architecture for future public wireless access," IEEE Communications Magazine, vol. 41, no. 3, pp. 108-113, 2003.

[4] H.-M. Chen, J.-B. Wang, and M. Chen, "Letter transmission systems outage performance of distributed antenna systems over shadowed nakagami-m fading channels," European Transactions on Telecommunications, vol. 20, no. 5, pp. 531-535, 2009.
[5] H.-M. Chen, C. Zhang, and M. Chen, "Outage probability analysis of the downlink ST-MRC distributed antenna system over shadowed Rayleigh fading channels," in Proceedings of the 5th International Conference on Wireless Communications, Networking and Mobile Computing (WiCOM '09), pp. 1-4, Beijing, China, September 2009.

[6] F. Héliot, R. Hoshyar, and R. Tafazolli, "An accurate closed-form approximation of the distributed MIMO outage probability," IEEE Transactions on Wireless Communications, vol. 10, no. 1, pp. 5-11, 2011.

[7] H.-M. Chen and M. Chen, "Capacity of the distributed antenna systems over shadowed fading channels," in Proceedings of the IEEE 69th Vehicular Technology Conference (VTC '09), pp. 1-4, IEEE, April 2009.

[8] D. Divsalar and M. K. Simon, "Improved parallel interference cancellation for CDMA," IEEE Transactions on Communications, vol. 46, no. 2, pp. 258-268, 1998.

[9] M. Chen, Y. Li, S. Cheng, and H. Wang, "On the bit estimators of partial parallel interference cancellation for DS-CDMA," in Proceedings of the International Conference on Communications (ICC '01), vol. 6, pp. 1945-1949, June 2000.

[10] T. K. Y. Lo, "Maximum ratio transmission," IEEE Transactions on Communications, vol. 47, no. 10, pp. 1458-1461, 1999.

[11] D. Wang, X. You, J. Wang, Y. Wang, and X. Hou, "Spectral efficiency of distributed MIMO cellular systems in a composite fading channel," in Proceedings of the IEEE International Conference on Communications (ICC '08), pp. 1259-1264, May 2008.

[12] Z. Chen, J. Yuan, and B. Vucetic, "Analysis of transmit antenna selection/maximal-ratio combining in Rayleigh fading channels," IEEE Transactions on Vehicular Technology, vol. 54, no. 4, pp. 1312-1321, 2005.

[13] M. K. Simon and M. S. Alouini, Digital Communication over Fading Channels, Wiley, New York, NY, USA, 2nd edition, 2005.

[14] A. Goldsmith, Wireless Communications, Cambridge University Press, New York, NY, USA, 2005.

[15] I. S. Gradshteyn and I. M. Ryzhik, Table of Integrals, Series and Products, Academic Press, San Diego, Calif, USA, 7th edition, 2007.

[16] G. L. Stuber, Principles of Mobile Communication, Kluwer Academic Publishers, 2nd edition, 1996.

[17] M. Abramowitz and I. A. Stegun, Handbook of Mathematical Functions with Formulas, Graphs, and Mathematical Tables, Dover Publications, New York, NY, USA, 9th edition, 1970.

[18] L. Jianjun, K. Hojin, K. Sungjin, and B. L. Kwang, "A new cell structure for distributed wireless communication system without inter-cell interference," in Proceedings of the IEEE Global Telecommunications Conference (GLOBECOM '05), pp. 2485-2489, St. Louis, Mo, USA, December 2005.

[19] R. L. Burden and J. D. Faires, Numerical Analysis, PWS KENT Publishing Company, Boston, Mass, USA, 7th edition, 1989.

[20] M. J. Ho and G. Stuber, "Co-channel interference of microcellular systems on shadowed Nakagami fading channels," in Proceedings of the 43rd IEEE Vehicular Technology Conference, pp. 568-571, May 1993. 


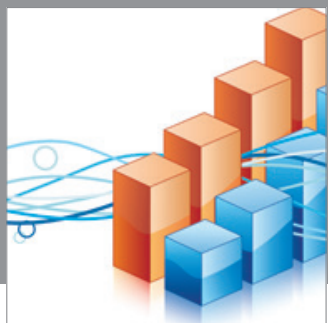

Advances in

Operations Research

mansans

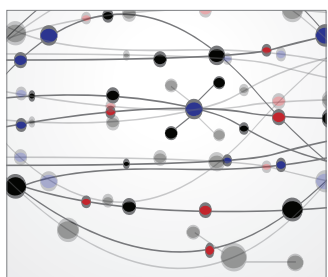

The Scientific World Journal
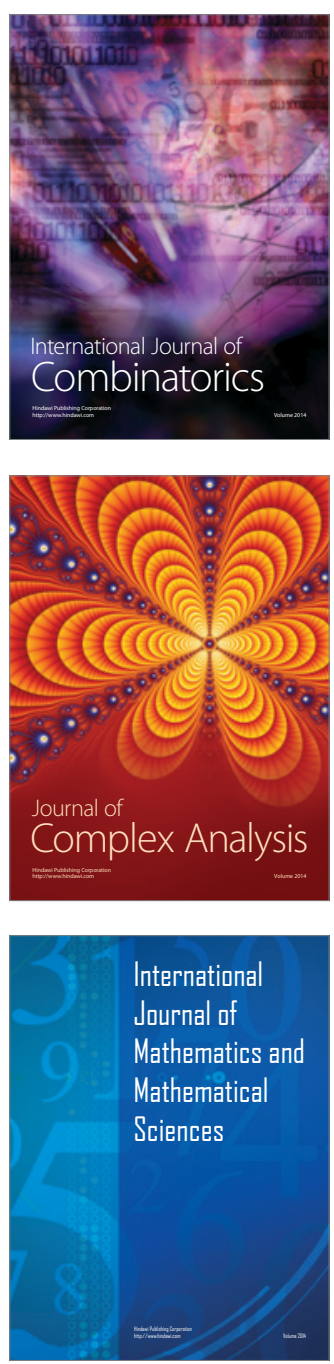
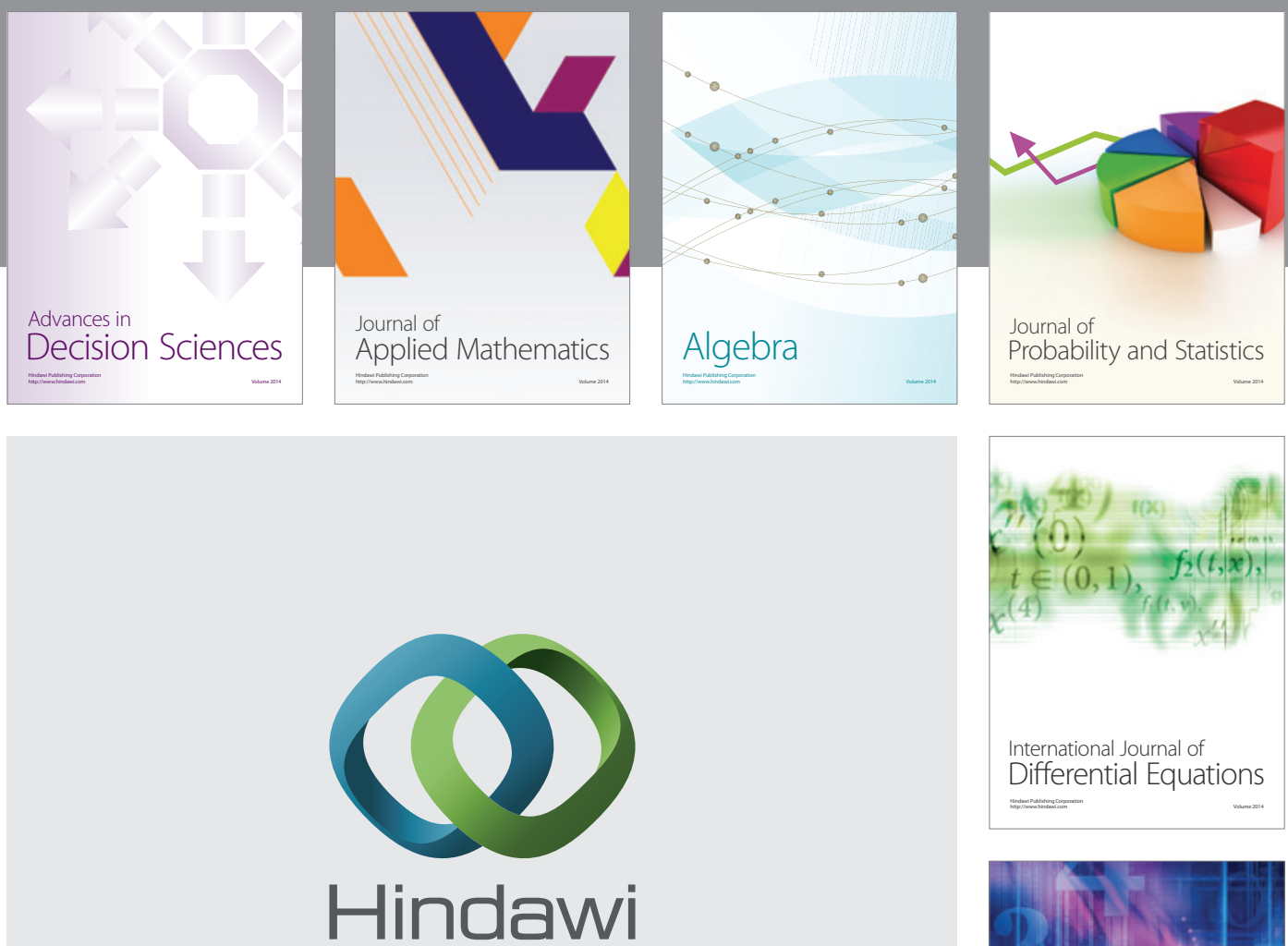

Submit your manuscripts at http://www.hindawi.com
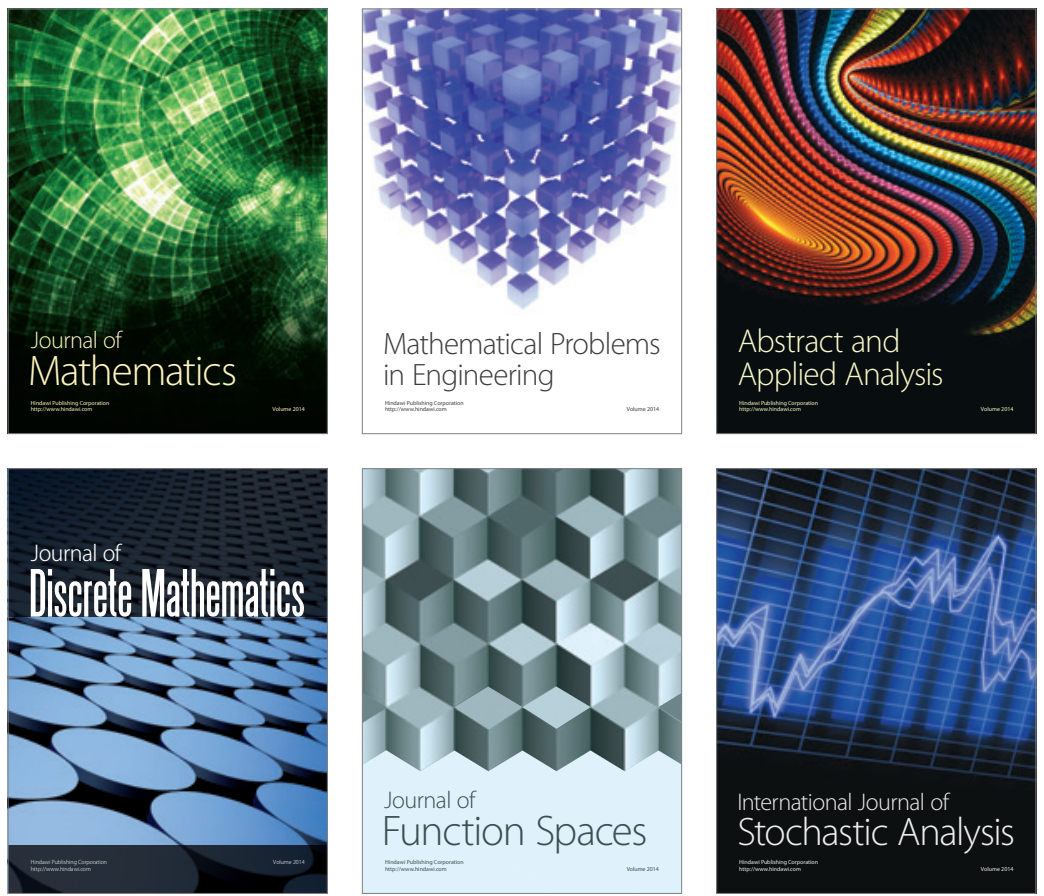

Journal of

Function Spaces

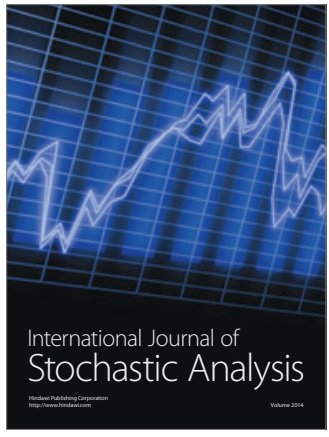

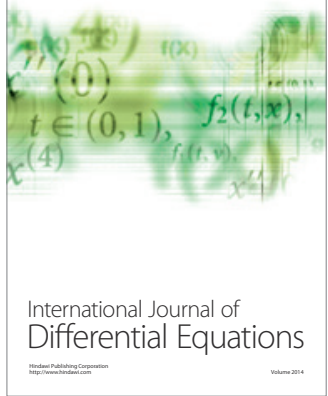
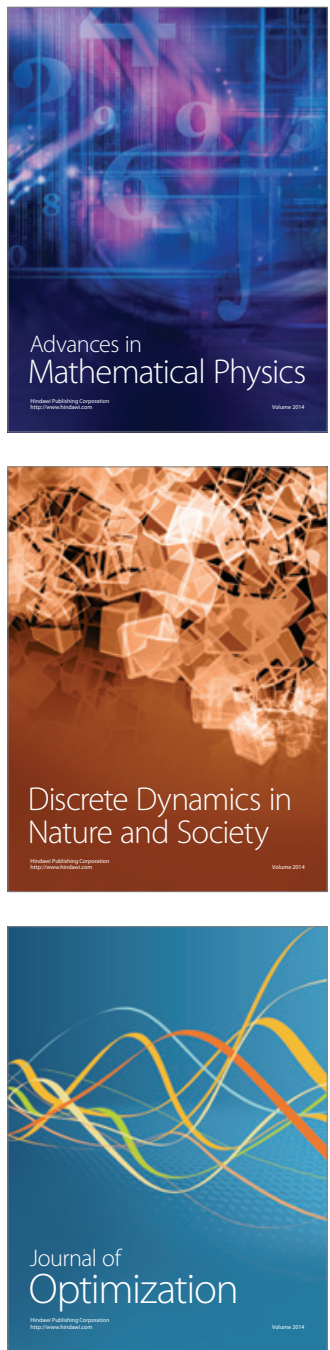